\title{
MORPHOLOGIES IN MPC-SIZE RADIO GALAXIES
}

L. SARIPALLI ${ }^{1,4}$, R. SUBRAHMANYAN ${ }^{2,4}$, R. W. HUNSTEAD ${ }^{3}$

${ }^{1}$ Indian Institute of Astrophysics, Koramangala,Bangalore 560 034, India

2 Raman Research Institute, Sadashivanagar, Bangalore 560 080, India

3 Astrophysics Department, School of Physics, University of Sydney, NSW 2006, Australia

4 Australia Telescope National Facility, CSIRO, P O Box 76, Epping, NSW 2121, Australia

\section{Introduction}

The extended radio structures or lobes found in edge-brightened radio galaxies represent interactions with the ambient medium over the source lifetimes. Their study probes the temporal evolution in the radio sources and the properties of the ambient medium encountered at different locations. Previous studies (Leahy \& Williams, 1984; Leahy et al., 1989) involved radio galaxies with sizes $\sim 400 \mathrm{kpc}$, and revealed radio morphologies with a variety of off-axis distortions that could be interpreted as due to different ways in which the lobes interact with the galactic halos. Such studies are lacking for radio galaxies having Megaparsec sizes, which extend to distances well beyond the observed galactic halos and are suspected of evolving in a different regime (Baldwin, 1982). Towards learning the evolution of radio galaxies in this size regime, we have carried out a study of a complete sample of Mpc-size radio galaxies; details are presented in Subrahmanyan, Saripalli \& Hunstead (to appear in MNRAS, 1996).

\section{Sample Selection}

Our sample of eight Mpc-size radio galaxies was selected from the MRC extended source sample of Jones \& McAdam, 1992. This sample is complete 
for sources with $S_{843}>1 \mathrm{Jy}$ and angular size $>1.5$ arcmin. We have further restricted the sample to sources with angular sizes in the range 3-12 arcmin to ensure that the imaging would reproduce all structural components in the sources. The selected sources are all edge-brightened with linear sizes $>750 h_{50}^{-1} \mathrm{kpc}$ and not located in any rich clusters or crowded fields. The sources were observed with the Australia Telescope at $1.4 \mathrm{GHz}$ and imaged with a resolution of 10 arcsec.

\section{Results and Discussion}

We have compared the radio-lobe morphologies in our sample of eight giants with the lobe morphologies in smaller-sized radio galaxies (the sample of Leahy \& Williams 1984) that have the same power and redshift range.

1. Sharp lobe boundaries are frequently seen in the Mpc-size radio galaxies. This indicates that the lobe plasma is confined and not diffusing freely into the ambient medium.

2. The axial ratios of the giants are found to be the same as the axial ratios in the smaller radio galaxies. The Mpc-size structures are simply scaled versions of smaller radio galaxies. This result is indicative of selfsimilarity in the evolution of radio galaxies.

3 The lobe off-axis distortions, that are frequently seen in small-size radio galaxies, are also present in the giants and are similar; however, the deviations from axial symmetry in the giants are seen to occur at distances as far as $500 \mathrm{kpc}$. This points to galaxy halos whose properties continuously fall with distance, as against having abrupt cutoff at sizes of $\approx 100 \mathrm{kpc}-$ the seen boundaries of the halo X-ray emission.

4. The lobes in the Mpc-size radio galaxies appear more fragmented and non-uniform except in cases where the neighbouring galaxy density is higher. The minimum pressures in the lobes are a factor seven lower than in the smaller sources, somewhat higher than expected considering the similarity in the axial ratios. This indicates smaller lobe filling factors for the giants.

5. A number of large galaxies show indications for discontinuous activity in the past: recessed hotspots, minima just upstream from the hotspots, and symmetrically-located, recessed enhanced emission regions.

\section{References}

Baldwin J.E., 1982, in Andernach H., Wielebinski R., eds, Proc. IAU Symp. 97, Extragalactic Radio Sources, Reidel, Dordrecht.

Jones P.A., McAdam W.B., 1992, ApJS, 80, 137.

Leahy J.P., Williams A.G., 1984, MNRAS, $210,929$.

Leahy J.P., Muxlow T.W.B., Stephens P.W., 1989, MNRAS, 239, 401. 